\title{
Comparison between Eulerian diagnostics and finite-size Lyapunov exponents computed from altimetry in the Algerian basin
}

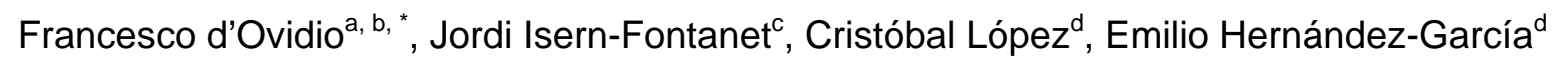 \\ and Emilio García-Ladona ${ }^{\mathrm{e}}$
}

\footnotetext{
a Laboratoire d'Océanographie et du Climat: Expérimentation et Approches Numériques, IPSL, Paris, France

${ }^{\mathrm{b}}$ Institute of Complex Systems, Paris Ile-de-France (ISC-PIF), France

c Laboratoire d'Océanographie Spatiale, Ifremer, Plouzané, France

d Instituto de Física Interdisciplinar y Sistemas Complejos IFISC (CSIC-UIB), Campus Universitat de les IIles Balears, E-07122 Palma de Mallorca, Spain

e Institut de Ciències del Mar, CSIC, Passeig Marítim de la Barceloneta 37-43, E-08003 Barcelona, Spain
}

*: Corresponding author : Francesco d'Ovidio, email address : $\underline{\text { dovidio@lmd.ens.fr }}$

\begin{abstract}
:
Transport and mixing properties of surface currents can be detected from altimetric data by both Eulerian and Lagrangian diagnostics. In contrast with Eulerian diagnostics, Lagrangian tools like the local Lyapunov exponents have the advantage of exploiting both spatial and temporal variability of the velocity field and are in principle able to unveil subgrid filaments generated by chaotic stirring. However, one may wonder whether this theoretical advantage is of practical interest in real-data, mesoscale and sub-mesoscale analysis, because of the uncertainties and resolution of altimetric products, and the non-passive nature of biogeochemical tracers. Here we compare the ability of standard Eulerian diagnostics and the finite-size Lyapunov exponent in detecting instantaneous and climatological transport and mixing properties in the south-western Mediterranean. By comparing with sea-surface temperature patterns, we find that the two approaches provide similar results for slowly evolving eddies like the first Alboran gyre. However, the Lyapunov exponent is also able to predict the (sub-)mesoscale filamentary processes occurring along the Algerian current and above the Balearic Abyssal Plain. Such filaments are also observed, with some mismatch, in sea-surface temperature patterns. Climatologies of Lyapunov exponents do not show any compact relation with other Eulerian diagnostics, unveiling a different
\end{abstract}


structure even at the basin scale. We conclude that filamentation dynamics can be detected by reprocessing available altimetric data with Lagrangian tools, giving insight into (sub-)mesoscale stirring processes relevant to tracer observations and complementing traditional Eulerian diagnostics.

Key words: Submesoscale, filaments, altimetry, Mediterranean circulation, Lagrangian dynamics

\section{Introduction}

Satellite high-resolution daily images of tracers like sea-surface temperature and chlorophyll show a large mesoscale and sub-mesoscale heterogeneity and patchiness, typically in the form of filaments. The full range of tracer variability observed in a climatological mean at the basin scale, can occur in daily snapshots over spatial scales of just 10$100 \mathrm{~km}$ (see for instance Lehahn et al. (2007) for the case of chlorophyll in the NE Atlantic). This large hetereogenity occurring on relatively short distances is able to induce very strong tracer gradients and to impact on important aspects of the ocean dynamics like lateral transport, upwelling/downwelling, and mixing. Mesoscale and sub-mesoscale variability is an important component of plankton dynamics (Abraham, 1998; López et al., 2001; Abraham et al., 2000; Boyd et al., 2000; Toner et al., 2003; Martin, 2003), larval transport (Bradburv and Snelgrove, 2001), as well as the dispersion of contaminants and oil spills. In polar regions, (sub-)mesoscale tracer variability has been recently recognised to impact also on the thermohaline properties of the mixed layer, affecting the low frequency ocean circulation through the formation of waters below the mixed layer (Sallee et al., 2006, 2007). Mesoscale and sub-mesoscale gradients of heat and salinity can induce cells of very strong vertical velocities (several tens of meters per day) from subsurface down to and below the mixed layer depth. These vertical velocities may fertilise the photic layer, creating local plankton blooms and affecting the sequestration of organic matter from the surface (Lévy et al., 2001, 2005). This mechanism can affect the properties of deep waters when they are formed (Paci et al., 2005). Tracer gradient intensification is also a precondition to mixing. Horizontally, the elongation of water masses in thin structures intensifies local gradients, greatly enhancing background diffusion (Lapeyre et al., 2006). Vertically, the trapping of inertial waves inside filaments creates mixing hotspots that extends to the bottom and below the mixed layer depth (Young et al., 1982).

Tracer mesoscale and sub-mesoscale patches are often found associated to surface mesoscale eddies (e.g. Robinson. A. R. et al. (1993); McGillicuddv et al. (1998); Martin et al. (2002); Abraham and Bowen (2002); Morrow et al. (2004); Legal et al. (2006)). Tracers are stretched into filaments by the shear-dominated regions in between mesoscale eddies while the recirculating regions inside eddies' cores can trap and transport tracer anomalies for timescales

* Corresponding author.

Email addresses: dovidio@lmd.ens.fr (F. d'Ovidio), jisern@ifremer.fr (Jordi Isern-Fontanet), clopez@ifisc.uib.es (Cristóbal López), emilio@ifisc.uib.es (Emilio Hernández-García), emilio@cmima.csic.es (Emilio García-Ladona). 
comparable to the eddy lifetime. As a well-known tool to reveal geostrophic velocities associated to eddies, altimetry data when opportunly analyzed, provide an important source of information for the mesoscale and sub-mesoscale structuration of tracer distributions.

Traditionally, transport information has been inferred from altimetry with Eulerian diagnostics like the eddy kinetic energy, the Okubo-Weiss criterion that separates the eddy's core from the periphery and others, that will be discussed in detail shortly. These diagnostics are based on the analysis of instantaneous snapshots of tracer and velocity fields. For instance, the Okubo-Weiss (OW) parameter has been applied to drifters in order to characterize eddy properties (Stocker and Imberger, 2003; Testor and Gascard, 2005), to in situ density profiles to identify regions with different mixing properties (Isern-Fontanet et al., 2004), and to altimetric maps with similar objectives (Isern-Fontanet et al., 2004; Waugh et al., 2006). The OW parameter has also widely been used for the identification and characterization of ocean eddies from altimetry (Isern-Fontanet et al., 2003; Morrow et al., 2004; Chaigneau and Pizarro, 2005; Isern-Fontanet et al., 2006). More recently, it has been suggested that Lagrangian diagnostics are more appropriate to link turbulence properties to tracer dynamics. The most used Lagrangian diagnostics have been the calculation of local Lyapunov exponents, that measure the relative dispersion of advected particles. In contrast to Eulerian tools, Lagrangian diagnostics do not analyze instantaneous snapshots of the velocity field, but measure transport properties along particle trajectories, therefore reconstructing the fine structure of transport dynamics that a fluid parcel has experienced, like subgrid filament formation. These patterns depend on the advection history along a trajectory, that may span a large spatiotemporal domain of the velocity field, and therefore cannot be captured by Eulerian diagnostics, that instead measure local, properties. Regarding Lagrangian tools like the finite-size or finite-time Lyapunov exponents (resp. FSLEs and FTLEs) Abraham and Bowen (2002) computed FTLEs from time-dependent velocity fields and compared to istantaneous SST patterns, LaCasce and Ohlmann (2003) used FSLEs to study the statistics of a large data set of drifting buoys; Waugh et al. (2006) applied FTLEs to altimetry and compared the results with OW and kinetic energy estimations; Lehahn et al. (2007) computed FSLEs from the geostrophic field in order to extract transport barriers and compared with satellite derived plankton and SST patches; Rossi et al. (2008) compared mixing and productivity by using the FSLE. Besides these studies, the FSLE has been also applied to numerical simulations of the Mediterranean sea with different objectives: the predictability of Lagrangian trajectories (Iudicone et al., 2002) and the characterization of flow patterns (d'Ovidio et al., 2004) and dispersion (García-Olivares et al., 2005).

However, for the case of real velocity data and given the chaotic nature of mesoscale advection (in the sense of positive Lyapunov exponents) one may expect Lagrangian diagnostics to be more affected than Eulerian tools by any space and time indetermination of the velocity field. When dealing with real altimetric data, therefore one may ask whether the theoretical advantage of Lagrangian techniques of detecting small scale transport structure is not countered in practice by the intrinsic error and cutoff of altimetric data, so that this information is either unreliable or already contained in Eulerian quantities. This question is particularly relevant given the larger computational costs (a few orders of magnitude) of Lagrangian tools in respect to Eulerian ones. In fact, for the case of the Tasman sea Waugh et al. (2006) found a striking resemblance between maps of Lyapunov exponents and the eddy kinetic energy, as well as a compact relationship between the two quantities. In their concluding remarks, Waugh et al. (2006) argued that this observation, if valid in other oceanic basins as well, would rise the possibility of using the EKE for estimating stirring rates, without the need of explicit, longer Lagrangian calculations. However, one of the main advantages of the Lagrangian techniques is the possibility of reconstructing tracer patterns that are below the resolution of the velocity field and that arise due to several iterations of stretching and folding during the tracer (chaotic) advection. Indeed, in 
Waugh et al. (2006) the correlation between the Lyapunov exponents have been found on a grid at relatively low resolution ( 0.5 deg.) and for a advection times of 14 days (two altimetric images), i.e. without the spatial and temporal information needed for the developement of filaments. Does this result hold also for filament-resolving Lagrangian calculations?

In this paper we address this question, exploring the possibility of obtaining reliable information with Lyapunov exponents that is not already contained in Eulerian diagnostics, focusing on filament dynamics.

This will be done both on the analysis of individual patterns (validated by high resolution SST images) and on a climatological basis. We find that at (sub-)mesoscale resolution the information provided by Eulerian diagnostics and Lyapunov exponents coincindes only for very stationary eddies, while providing two distinct and complementary pictures of the circulation in all the other cases: the Eulerian analysis provides the eddies that populate the mesoscale, while the Lagrangian analysis yields the tracer filaments generated by the spatiotemporal variability of these eddies. Our results also show a surprising reliability of altimetric data at the scale of their nominal resolution, when reprocessed with Lagrangian tools.

We will compare Eulerian diagnostics and Lyapunov exponents in the Algerian basin because of its rich and variable mesoscale activity, that contains jet-dominated regions (the path of the Algerian current) as well as eddies with very different characteristics (from the quasistationary Alboran gyres, to the slowly propagating eddies of the Balearic Abyssal plain, and the fast-evolving Algerian eddies). A main characteristic of the surface circulation in the Mediterranean sea is the propagation of fresh waters incoming from the Atlantic ocean. At the entrance of the Mediterranean, these waters flow from west to east and form patterns such as the Alboran eddies east of the Gibraltar strait or the Algerian current along the Algerian coast. The instabilities of this current generates, a few times per year, coastal eddies that propagate downstream, usually until the entrance of the Sardinia channel. There, they can detach from the coast and propagate as open sea eddies (Millot, 1999) following relatively well defined paths (Isern-Fontanet et al., 2006). These eddies, called Algerian eddies, have variable diameters of about $50-200 \mathrm{~km}$, vertical extents from hundreds to thousands of meters, and lifetimes of several months, up to nearly 3 years (Millot et al., 1997; Puillat et al., 2002). Their presence has a large impact on the redistribution of tracers in the Algerian basin, which is characterized by the northward spreading of tracers that are initially transported eastwards by the Algerian current (Ovchinikov, 1966; Brasseur et al., 1996). As we shall see, our analysis of stirring will unveil the crucial role on tracer patterns of the time variability of the mesoscale activity in the Algerian basin and the relevance of topography in constraining the dynamics of coherent structures.

The paper is organized as follows. After describing the data sets and the techniques, we develop an Eulerian and Lagrangian analysis for specific days. We focus on the representation of eddies and the detection of transport barriers and we get two complementary pictures: a regular and smooth OW-based description and a more complex, lobular representation from the FSLE. We then compare such different structures to tracer distributions. In order to filter out the active dynamics of a real tracer as well as the unresolved components and indeterminacies of altimetry data, we first consider the filaments of a synthetic tracer, that we advect numerically with the altimetric data. As a second step, we take sea-surface temperature (SST) satellite images. The indications that are found on the individual days are then generalized in a climatological comparison, where we compare the spatial variability of FSLE, OW parameter, strain rate, and eddy kinetic energy temporally averaged over the period 1994-2004. For both the Eulerian and the Lagrangian analysis, we also propose to describe the consequences of filament formation with a climatology giving the spatial density of transport barriers. 


\section{Methods}

A traditional approach to the characterization of the stirring and mixing in the presence of mesoscale eddies consists in separating the stagnation region at the eddy's core from the eddy's periphery where tracer filamentation occurrs. This is done by measuring the relative dominance of vorticity and deformation. One of the most used parameters for measuring this relative dominance is the Okubo-Weiss parameter (Okubo, 1970; Weiss, 1991) which has been already used by some of us to study properties of Algerian eddies (Isern-Fontanet et al., 2004). The OW parameter is a particular case of the more general vortex-identification criterion proposed by Jeong and Hussain (1995).

The Okubo-Weiss parameter $W$ is defined as:

$$
W=s_{n}^{2}+s_{s}^{2}-\omega^{2}
$$

where $s_{n}, s_{s}$ and $\omega$ are the normal and the shear components of strain, and the relative vorticity of the flow defined, respectively, by

$$
s_{n}=\frac{\partial u}{\partial x}-\frac{\partial v}{\partial y}, \quad s_{s}=\frac{\partial v}{\partial x}+\frac{\partial u}{\partial y}, \quad \omega=\frac{\partial v}{\partial x}-\frac{\partial u}{\partial y} .
$$

In the formula above, $x$ and $y$ are orthogonal spatial coordinates and $u$ and $v$ are the component of the velocity respectively for the $x$ and $y$ directions.

The parameter $W$, allows to separate a two-dimensional flow into different regions: a vorticity-dominated region $\left(W<-W_{0}\right)$, a strain-dominated region $\left(W>W_{0}\right)$ and a background field with small positive and negative values of $W\left(|W| \leq W_{0}\right)$. Here $W_{0}=$ $0.2 \sigma_{W}, \sigma_{W}$ being the standard deviation of the $W$ values in the whole domain, in our case the Mediterranean sea (Bracco et al., 2000; Pasquero et al., 2001; Isern-Fontanet et al., 2006; Elhmaïdi et al., 1993). The core edge can then be identified as the closed lines with $W=0$. This separation of the field in terms of the sign of $W$ has been proved to be a robust criterion for extracting eddy cores from complex fluid flows (Jeong and Hussain, 1995; Pasquero et al., 2001). In steady flows, the boundary of the core constitutes a barrier to the exchange of particles with the surrounding cell, so that particles trapped inside the eddy core remain there. Under non-steady flows, particles can leak out of the vortices, be ejected through filamentation processes, or even the eddy can be destroyed (Basdevant and Philipovitch, 1994; Hua and Klein, 1998).

The OW parameter has some well-known limitations. On one side, it assumes that velocity gradients are slowly evolving in time, which is only valid inside relatively coherent regions. On the other side, this parameter fails to properly identify regions with different mixing properties when eddies are stationary and have axial symmetry (Lapeyre et al., 1999). There have been some attempts to solve some of these limitations by extending it to consider the time evolution of velocity gradients (Hua and Klein, 1998; Hua et al., 1998; Lapevre et al., 1999).

Another approach to the characterization of flow structures is to assume a Lagrangian viewpoint, that is, to look explicitly at transport properties from the analysis of particle trajectories (Pierrehumbert, 1991; Ridderinkhof and Zimmerman, 1992; Miller et al., 1997;

Haller and Yuan, 2000; Coulliette and Wiggins, 2001; Koh and Legras, 2002; d'Ovidio et al., 2004; Shadden et al., 2005; Mancho et al., 2006). In contrast to the OW method and in 
general to Eulerian diagnostics, this approach requires knowledge of the time variability of the velocity field, as well as the use of an integrator for generating the trajectories. Several methods exist, one of the simplest being the Finite-Size Lyapunov Exponent (FSLE). The FSLE is a generalization of the concept of Lyapunov exponent to finite separations. The standard definition of Lyapunov exponent refers to the exponential rate of divergence, averaged over infinite time, of infinitesimally closed initial points. The FSLE technique keeps the original idea of capturing the rate of divergence between trajectories, but overcomes the limit operations. Thus, it is (and has been shown to be) rather appropriate to manage real data. The FSLEs were introduced for turbulent flows (Aurell et al., 1997; Artale et al., 1997) aiming at studying non-asymptotic dispersion processes. Since then, they have been used for two complementary goals: for characterizing dispersion processes (Lacorata et al., 2001), and for detecting and visualizing Lagrangian structures (e.g. transport barriers and fronts) (Boffetta et al., 2001; Koh and Legras, 2002; Joseph and Legras, 2002; d'Ovidio et al., 2004). In the framework of this paper, in order to compare with the OW parameter we will focus mainly on the second use.

Several methods allow to calculate the FSLEs. In the simplest scheme, for each instant $t$ and each point $\mathbf{x}$, one follows in time the evolution of a tracer started in $\mathbf{x}$ and of another probing tracer located at a distance $\delta_{0}$ from it. The integration is stopped when the two tracers have reached a final separation $\delta_{f}>\delta_{0}$. From the time interval, $\tau$, to reach the final separation, the FSLE is defined in the following way:

$$
\lambda\left(\mathbf{x}, t, \delta_{0}, \delta_{f}\right)=\frac{1}{\tau} \log \frac{\delta_{f}}{\delta_{0}} .
$$

In order to reduce the dependence on the direction of the probing tracer, the algorithm is run choosing three points forming an equilateral triangle around $\mathbf{x}$. We stopped the integration when any of these three points reaches a separation $\delta_{f}$ from the trajectory started in $\mathbf{x}$.

Maxima (ridges) of Lyapunov values are typically organized in convoluted, lobular lines. For the case of the backward calculations, these lines can be interpreted as the fronts of passively advected tracers. This interpretation can be understood in a very qualitative but effective way, considering that fronts typically separate fluid patches of different origins. Therefore, the separation in the past of a couple of points is largest when the couple is initialised exactly over the front. This argument can be rephrased in a slightly more rigorous way in the context of dynamical systems, interpreting the line-shaped regions of fastest separation -either backward or forward in time- respectively as the unstable and stable manifolds of the hyperbolic points in the flow (Haller and Yuan, 2000; Boffetta et al., 2001; Haller, 2001; Koh and Legras, 2002; Joseph and Legras, 2002; d'Ovidio et al., 2004; Mancho et al., 2006). The effect on advection of hyperbolic structures is sketched in Fig. 1 Due to the convergent field along the stable manifold and the divergent field along the unstable manifold, a passively advected tracer is deformed as in Fig. 1. developing a front along the unstable manifold and a gradient orthogonally to it. Due to the hyperbolic structure of which the manifold is a part, the tracer front approaches the manifold exponentially fast. The distance $\delta_{r}$ between the tracer front and the manifold depends on the initial front-to-manifold distance $\delta_{i}$, the exponent $\lambda$ of the manifold, and the time of integration $t$ :

$$
\delta_{r} \approx \delta_{i} \exp (-\lambda t) .
$$

Manifolds characterized by higher exponents have therefore a stronger effect on tracers, shaping a front in shorter times and being more visible in tracer distributions. 
Note that for a time-dependent velocity field, the sketch of Fig. 1 1holds only if the hyperbolic structures evolve in time on a time scale slower than the tracer advection, so that the tracer can actually relax over the manifold. See Lehahn et al. (2007) for more details on the FSLE computation from altimetric data.

Mixing properties can also be diagnosed by Lyapunov exponent calculations, either considering the exponential separation in the future as a measure of tracer dispersion, or by combining forward and backward information (d'Ovidio et al., 2004). Since in this work we aim at a direct comparison with advected tracers (SST), we will focus on the backward calculation and compare the location of the manifolds detected by the FSLEs with tracer fronts.

As it is clear from Eq.(2), the FSLEs depend critically on the choice of two length scales: the initial separation $\delta_{0}$ and the final one $\delta_{f}$. d'Ovidio et al. (2004) argued that $\delta_{0}$ has to be close to the intergrid spacing $\Delta x$ among the points $\mathbf{x}$ on which the FSLEs will be computed. In fact, a $\delta_{0}$ larger than intergrid spacing would allow to sample manifolds of strong Lyapunov exponents in more than one grid points, while $\delta_{0}$ smaller than the intergrid spacing would not allow to follow a manifold on the sampling grid as a continuous line. Following d'Ovidio et al. (2004); Lehahn et al. (2007), in this work we have set $\delta_{0}=0.01^{\circ}$ (approx. $1 \mathrm{~km}$ ) in order to match the resolution of SST images. We have set $\delta_{f}=1^{\circ}$ i.e., separations of about $110 \mathrm{Km}$, that is the order of magnitude of the eddies' radii detected by altimetry. Values of $\delta_{f}$ smaller or larger up to $50 \%$ do not change significatively the calculation. The time of integration for finite-size Lyapunov exponents varies from one point of another, being small for strong values and vice versa. Inverting Eq2.

$$
\tau=\frac{1}{\lambda} \log \frac{\delta_{f}}{\delta_{0}}
$$

We found typical values of finite-size Lyapunov exponents in the range $0.1-0.2$ days $^{-1}$, corresponding to integration times of resp. 46 and 23 days.

\section{Data}

\subsection{Sea-Surface Height}

In the Mediterranean sea, despite the weak signal intensity and the coarse space and time resolution of the altimetric tracks, several studies have shown the reliability of the altimetric data to analyze its dynamics, particularly in the Algerian basin Vignudelli, 1997; Bouzinac et al., 1998; Larnicol et al., 2002; Font et al., 2004). In this study we have used Delayed Time Maps of Absolute Dynamic Heights (DT-MADT) produced by Collecte Localisation Satellites (CLS) in Toulouse (France) specifically for the Mediterranean sea, which combine the signals of ERS-ENVISAT and TOPEX/Poseidon-JASON altimeters.

Altimetric data are processed including usual corrections (sea-state bias, tides, inverse barometer, etc.) and improved orbits. From several corrected sea-surface height files, a conventional repeat-track analysis is performed to extract the Sea Level Anomaly (SLA) relative to a mean profile: data are re-sampled along the mean profile using cubic splines and differences relative to the mean profile are calculated. SLA along-track data are then 
filtered and subsampled. The filters used are a non linear median over 3 points (roughly 21 $\mathrm{km}$ ) followed by a low pass along track linear Lanczos filter (with a cut-off wavelength of 42 $\mathrm{km}$ ). SLA data are then subsampled every other point (SSALTO/DUACS User Handbook, 2006). Finally, SLA maps are built using an improved space/time objective analysis method, which takes into account long wavelength errors, on a regular grid (Le Traon et al., 1998) of $(1 / 8)^{\circ} \times(1 / 8)^{o}$ every week. Then, Sea-Surface Heights (SSH) are finally obtained by adding to the SLA a Mean Dynamic Topography (Rio et al., 2007).

For each data set geostrophic velocities are estimated as usual:

$$
u=-\frac{g}{f R_{T}} \frac{\partial h_{s s h}}{\partial \phi}, v=\frac{g}{f R_{T} \cos \phi} \frac{\partial h_{s s h}}{\partial \lambda},
$$

where $h_{s s h}$ is the SSH, g is gravity, $f$ the Coriolis parameter, $R_{T}$ the Earth radius, $\phi$ the latitude and $\lambda$ the longitude. The data analyzed spans from January 1, 1994 to December 31, 2004. From this data set we study representative days and we also construct climatologies. Finally, a Runge-Kutta integrator of fourth order and a time step of 6 hours has been used to obtain backward and forward trajectories in the velocity field for the calculation of FSLEs and for the advection of a synthetic tracer. The geostrophic velocity field has been resampled in space and time with a multilinear interpolator.

\subsection{Sea-surface temperature}

We used sea-surface temperature (SST) data from the AVHRR sensors on board NOAA satellites, downloaded from the HRPT station at the Institut de Ciències del Mar (CSIC) in Barcelona. For the single day analysis we have chosen images for which there were good quality sea-surface temperature images presenting a large variety of eddy structures and different intensities of the Algerian current: July 9, 2003; April 7, 2004; June 30, 2004. SST images have a resolution of $1.1 \mathrm{~km}$ at the nadir.

\section{Results}

\subsection{Eddy representation}

Figure 2 2 shows the spatial distribution of OW, which appears as a set of vorticity-dominated ( $W<0$, blue) regions surrounded by strain-dominated lobular structures $(W>0$, red $)$ embedded in a background field of small values of $W$. On the other hand, Fig. B shows the spatial distribution of FSLEs which has very different patterns characterized by a tangle of lines. These are the locations were FSLE are large, approximating unstable manifolds and corresponding to transport barriers embedded in a background field with $\lambda \simeq 0$. The unstable manifolds arise from fluid stretching and they are therefore Lagrangian analogs of the deformation OW regions.

A first important difference in daily maps of OW and FSLE values is the presence of eddies in the first and of filaments in the second. In particular, in the FSLE map there are no enclosed regions and in some cases (e.g. for the eddies over the Balearic abyssal plain), the 
same manifold connects multiple lobes, spanning a region of several eddies. When comparing these patterns with the stream-function of the flow, SSH in this case, it can be observed that in general eddies localized by the OW parameter and the centers of the spiralling FSLE lobes are in good agreement with the extrema of the altimetric field, as one would expect. Across the Algerian current, the OW field identifies some possibly spurious eddies that do not appear in either the FSLE or SSH. This is due to the fact that both SSH and FSLE are not invariant under a transformation of coordinates to a frame of reference moving at a constant velocity with respect to the original (Galilean transformation) and therefore, eddies are hidden or partially hidden by the presence of the Algerian current. Since OW is Galilean invariant it is able to detect these eddies but it fails in the detection of the Algerian current which appears as a coherent structure characterized by manifolds (barriers) parallel to the altimetric streamlines in the FSLE picture.

Another key difference between both fields is linked to the time-evolution of coherent structures. From Figs. 2 and 3 it can be seen that eddies displaying a similar size and intensity in the OW map may show very different features in the FSLE map. This is the case for instance for the western Alboran eddy and some of the mesoscale eddies in the Algerian basin (especially during July 30, 2004). For the Alboran vortex, the lobe spiral is very tight, almost resembling the concentric altimetry lines. For the eddies in the Algerian basin, the lobes are loose, much less localized, and discordant with the altimetric contours. It is interesting to notice that previous studies (e.g. Isern-Fontanet et al., 2006, and references therein) have shown that vortices in the Algerian basin propagate at velocities of the order of $5 \mathrm{~km} \mathrm{day^{-1 }}$ in contrast to the western Alboran eddy, which is almost stationary. In the ideal case of a time-independent velocity field, particle trajectories, as well as the set of lines in which Lyapunov exponents are organized (which approximate material lines), coincide with altimetric isolines. For the case of an eddy, they appear as concentric, closed manifolds. In a time-dependent flow, the identity between trajectories and altimetric isolines is lost and the differences quantify the time variability. The material lines resemble concentric circles for slowly evolving persistent structures like the Alboran gyres and assume a complex shape in the case of more dynamically active eddies, like the eddies along the Algerian current and some of the eddies over the Algerian basin. This phenomenon has an important effect on the transport properties. Due to the fact that material lines act as transport barriers, eddies with a low time variability and concentric unstable manifolds have a smaller water mass exchange with the surrounding compared to dynamical active vortices (see Lehahn et al. (2007) for a discussion of the eddy time variability in connection with phytoplankton pattern formation).

A third important difference between the OW and FSLE approaches is the spatial scale of the structures detected. The OW parameter is bounded by the resolution of the altimetric data $\left(1 / 8^{\circ}\right)$ and the low-pass filters applied during the construction of altimetric maps. This limitation does not hold for the FSLE that is based on trajectory calculations, whose length scale is a combination of both space and time variability of the velocity field. Lobes and filaments below the altimetric resolution appear, especially in the more dynamically active regions, like the fast evolving eddies formed downstream of the Algerian current or the lobes south of the Balearic islands. Very thin filaments are also associated with the Algerian current. 


\subsection{Detection of tracer fronts}

\subsubsection{Synthetic tracer}

First, we test the ability of OW and FSLE to characterize tracer distribution in an ideal but realistic situation: three sets of particles are advected by the velocity field estimated from altimetry. To this end we put three sets of particles distributed on a square grid centered over three different dynamical structures: the slowly evolving Alboran eddy, the Algerian current, and the strongly interacting eddies in the easternmost part of the Algerian basin (dashed boxes in Fig. 4). Particles in the eddy regions have been advected for two weeks. A shorter advection time of one week has been used for the particles initially placed over the Algerian current, due to the strongest velocity field in this region. The advection time that we have chosen is such that the particles are kept close to the dynamical structures we want to study: a larger advection time does not change the results discussed, but increases the dispersal of the particles over several structures.

The tracer released over the west Alboran eddy shows a regular, circular pattern well correlated with both the OW and the FSLE maps. This pattern differs from the more deformed distribution for the tracer released over the Algerian basin. In this region, the tracer appears spread on several eddies connected through thin filamentary structures. The boundaries detected by the OW parameter (we plot in Fig. 4, top, the lines $W=0$ ) provide an approximate picture, often underestimating the size of the eddy cores and providing no information of the patterns followed by the tracer exchanged from one eddy to another. In contrast, the FSLE lines of intense stretching reproduce with remarkable accuracy the tracer eddy boundaries, as well as the tangle of spiralling filaments that connect them. This is seen in Fig. 4 (bottom) where we plot the regions where the FSLE has values larger than $0.2 \mathrm{day}^{-1}$. As discussed above, these regions are essentially one-dimensional lines. They behave as material unstable manifolds of the advecting flow: they are almost perfectly located along the tracer boundary. Small deviations may be attributed to different reasons: (i) a tracer front approaches the manifold exponentially fast, so that a residual distance remains for a finite time of integration; (ii) only the manifolds with an intensity larger than 0.2 day $^{-1}$ have been plotted and other weaker lines may also act as transient transport barriers. The residual distance from a manifold can be estimated by Eq. 3: considering an initial tracer to manifold distance of about $100 \mathrm{~km}$, an exponent of $0.2-0.3 \mathrm{day}^{-1}$ for the manifold (Fig. 3), and an advection time of 15 days, we get a separation between the manifold and the tracer front of a few $\mathrm{km}$. Note that the thin filament at $7^{\circ} \mathrm{W}, 37.5^{\circ} \mathrm{N}$ is below the altimetric resolution, and appears in both the tracer advection and the FSLE map.

Thin filaments also appear for the case of the tracer released over the Algerian current. The OW is designed to detect vortices and therefore its use for barrier detection along the Algerian current is, strictly speaking, improper. Nevertheless, the gyre due to the mesoscale eddy located at $0.5^{\circ} \mathrm{W}, 36.5^{\circ} \mathrm{N}$ is correctly predicted. Not surprisingly, features due to smaller and rapidly evolving eddies (like the ones located along the current), as well as the barrier effect due to the current itself, are not detected. Interestingly, an OW signal appears in correspondence to the Almeria-Oran front (well visible in the southern boundary of the tracer), probably as a signature of the secondary Alboran eddy. Such a signature is composed of broken and irregular structures, but nevertheless is in phase with the southern front of the tracer. The OW does not provide any indication of the filament intruding the Algerian basin. In contrast with the lobular structures detected for eddies, the FSLE shows for the Algerian current meandering lines that follow the African coast. Such lines are in almost perfect agreement with the tracer distribution, well indicating the region of intrusion in 
the Algerian basin. A spiralling lobe at the location of the eddy also detected by the OW method at $0.5^{\circ} \mathrm{W}, 36.5^{\circ} \mathrm{N}$ is correctly localized. The Almeria-Oran front is well detected by a manifold that follows uninterrupted the Algerian current, marking a transport barrier that confines the water masses coming from the Alboran sea to an isolated tongue along the African coast. In agreement with this picture, the tracer has no intrusion with such coastal water, being initialized northern to such a manifold.

\subsubsection{An observed tracer: $S S T$}

Figure 5 depicts the temperature distribution for the three days selected and Fig. 6 shows some zooms corresponding to the dashed squares in Figs. 2, 3 and 5 ,

Figure 6 6 shows the situation of a relatively isolated westward propagating Algerian vortex. The center of the eddy is well located by the OW parameter, but the strongest SST gradients are beyond the outside of the $W=0$ which identify the vortex core. FSLE lines reproduce the two-lobe structure of the SST positive anomaly contered in $1 \mathrm{E}, 37.5 \mathrm{~N}$, although the size is overestimated. Furthermore, south of this vortex there is a filament of colder waters that approximately follows the transport barriers depicted by FSLE. However, this example also shows one of the limitations of the approach: FSLE and OW strongly depend on the quality of altimetric maps. A coastal eddy in the Algerian coast is clearly observable in the SST images but is not properly captured by SSH, and therefore partially missed by OW and FSLE.

Figure 6b depicts the Alboran sea in spring (April 7, 2004). In its westernmost part, close to the Gibraltar strait, a water mass of warm waters, surrounded by colder waters, is trapped within the western Alboran gyre. Both the OW and the FSLE maps show a regular and circular barrier although both seem to underestimate the radius of the object, maybe due to the location of the vortex in altimetric data. The Almeria-Oran front on the eastern part of the image is observable in both SST and FSLE but not in the OW field. Proceeding to west a large eddy attached to the coast is quite properly identified in all fields: SST, FSLE, $\mathrm{SSH}$ and OW. However, in the middle of the image, where the eastern Alboran eddy is usually located there is a poor coincidence between patterns calculated from altimetry and the SST image. In particular, the SST fronts observed at $3^{\circ} \mathrm{W}$ are almost perpendicular to the FSLE lines there.

Figure 6r shows an example of the strong signature of the Algerian current. In contrast with the previous case, the FSLE map reproduces with great accuracy the SST distribution. The dynamical barriers due to the presence of the jet along the coast are parallel to the SST isolines. In analogy to what was observed for the synthetic tracer, the jet has no signature on the OW parameter. On the other hand, several mesoscale eddy boundaries with no effect on the SST pattern also appear in the OW image. Centered at $37^{\circ} \mathrm{N} 2^{\circ} \mathrm{E}$ there is a large coastal eddy probably generated by the destabilization of the Algerian current. This eddy is clearly identified in all fields. However, the most remarkable pattern is the deflection of coastal waters from coast to the open sea due to the presence of this eddy, which is observable in FSLE as well as in SST. Note also that water masses in the inner part of the vortex are bounded by transport barriers and therefore trapped within the eddy.

Finally, Fig. 6 shows the easternmost part of the Algerian basin where several eddies strongly interact. Notice that this is one of the situations analyzed using the advection of ideal particles in the previous section. As expected, the match between real SST data and the FSLE is as good as before. The manifold tangle observed in the FSLE map can also be seen in the SST image and the cores of the eddies on which these manifolds wind are 
identified also by the OW parameter. An isolated region along the Algerian coast (7E, 37N), appearing in the SST image as a strong cold anomaly, is also fairly well identified by FSLE and not by the OW parameter.

\subsection{Time averaged fields}

In order to generalize the comparison performed on instantaneous cases, we focus now on the relationships between spatial field distributions averaged over time. To this end OW and FSLE have been computed for 10 years of data (1994-2004). The OW parameter is calculated for each altimetric image (at one week time resolution) while an FSLE map is generated each two days.

\subsubsection{Distribution of barrier-type lines}

First, we estimate the fraction of time during which each spatial point is visited by barriertype lines. By barrier-type lines we mean lines (ridges made of local maxima in FSLE and the $W=0$ isolines in OW) which could be interpreted, at least during some short time, as a transport barrier. To this end we count how many times each grid point pertains to one of such lines and divide by the total number of observations for that point (the length of the time-series). Figure 7 shows these local probabilities of having a transport barrier estimated using OW and FSLE (and denoted by $P_{W}$ and $P_{\lambda}$, respectively). In agreement to what we found in the previous sections, we observe that the pattern corresponding to OW parameter has a more regular, patchy structure due to the presence of Eulerian eddies, and no clear signature for features that are not dependent on individual vortices, like the Algerian current which appears instead in the FSLE map. This suggests the idea that features that are common in both maps might be associated to long-lived non-propagating vortices. The most evident example is the western Alboran eddy. Other examples are the pattern observed around longitude $4^{\circ} \mathrm{E}$ and $38^{\circ} \mathrm{N}$ which is associated to the region of vortex detachment from coast discussed in previous papers (Isern-Fontanet et al., 2006), the pattern observed east of Eivissa island $\left(1.5^{\circ} \mathrm{E}\right.$ and $\left.38.5^{\circ} \mathrm{N}\right)$ or the Almeria-Oran front.

After observing that OW and FSLE provides very different estimations of the propensity of the points to belong to a barrier-like line, the next question is to identify which one is closer to give a true barrier intensity and location. As outlined in the introduction, a characteristic of the incoming Atlantic waters with respect to the resident Mediterranean ones is their lower salinity. If there are significant barriers to the spread of this surface water into the Mediterranean, climatological distributions of salinity should locate them. Figure 8 shows the climatological salinity obtained from MEDATLAS-II data set. When comparing Figs. 7 and 8 it is evident that the estimation computed from FSLE provides a better picture, although the smoothness of the climatological salinity makes difficult the comparison. On Alboran, salinity increases eastwards. Close to the Almeria-Oran front the isohalines are almost aligned width the front, as depicted by FSLE. Eastwards, the intrusion of fresh waters along the coast following the Algerian current and the associated intense northwards gradient matches quite well the patterns observed in Fig. 7 (bottom). Proceeding to the east, at the entrance of the Sardinia channel FSLE barrier density is concentrated close to the coast, in correspondence with low salinity waters being also confined to this area. In the middle of the basin, the probability of having transport barriers is more homogeneous due to the propagation of Algerian eddies and consequently climatological salinity is more uniform. 


\subsubsection{Time averages of FSLE and other Eulerian diagnostics}

Results in previous sections suggest that high FSLEs are preferentially located on energetic structures like at the eddy periphery or along jets. This suggest a possible correlation between the FSLEs and other Eulerian diagnostics different from OW, such as the eddy kinetic energy (EKE) or the strain. In fact, in a recent study of the Tasman sea, it has been shown that averages of both the EKE and the strain have a compact relationship with averages of finite-time Lyapunov exponents (Waugh et al., 2006). Although such relationships are valid only on time average, and therefore cannot be used for the detection of instantaneous, local transport barriers, the correlation between EKE, strain, and Lyapunov exponents would provide a simple way to estimate Lyapunov exponent climatologies, as also suggested in Waugh et al. (2006). Finite-time and finite-size Lyapunov exponents only differ on the determination of the integration time (set a priori for finite-time, given indirectly by prescribing the final separation of the trajectories for finite-size). For this reason, we tested the hypothesis above by computing for our data period time averages of strain rate and of EKE.

Climatologies of FSLE, EKE, and strain rate are shown in Fig. 9. Similar structures appear in regions containing persistent objects, such as the Alboran gyre and the Algerian current. A large-scale gradient is also observed, with more signal in the south than in the north of the Algerian basin, and positive anomalies in the Sardinia channel. However, there are more localized mesoscale anomalies in the FSLE field.

In Fig. 10, joint distributions of FSLEs vs strain rate and EKE are presented. Although theres is a general positive correlation among these quantities, they show a much looser relationship than the one observed by (Waugh et al., 2006) at lower resolution and on shorter integration times. The lack of compact relationships on the scatter plots, as well as different distribution of anomalies among the various diagnostics is a clear confirmation that the Lyapunov exponent provide a complementary information in respect to Eulerian diagnostics even in a climatological sense, when the Lyapunov calculation is done at high resolution. The comparison with other Eulerian diagnostics (like the vorticity and the vorticity gradient, that is sometimes also used for transport barrier detection, see for instance (Paparella et al., 1997)) confirm these observations.

\section{Summary and discussion}

In this work we have exploited the rich mesoscale activity of the Algerian basin for comparing Eulerian and Lagrangian diagnostics of transport under various conditions. This comparison unveiled two coexisting pictures: an Eulerian view, dominated by mesoscale eddies, and a Lagrangian view, characterized by interconnected lobular structures and submesoscale filaments. The first picture describes the eddies that populate the mesoscale turbulence; the second one describes the spatial structures of tracers advected by these eddies. Eddy cores are usually identified by setting a threshold $(W=0)$ on the OW parameter, and this $W=0$ line may be a rough candidate to a 'transport barrier' since eddy contents are seen to remain coherent in several situations. More precise candidates to transport barriers are obtained from local maxima (ridges) of the FSLE computed backward in time.

As diagnostics of transport barriers, both techniques are based on heuristic arguments related to the time variability of the velocity field. The OW use is based on the consideration that for time-invariant fields, Lagrangian and Eulerian barriers coincide. For this reason, with the OW method no structure typical of time varying fields like filaments and lobular 
patterns can be detected but nevertheless remarkably good results can be obtained for stationary eddies, in our case the first Alboran gyre and some of the most persistent eddies over the Balearic abyssal plain. The FSLE calculation goes one step further, assuming quasi stationarity not for the velocity field, but for the lines of maximal divergence (unstable manifolds of hyperbolic points). When this time-scale separation between the Lagrangian structures and the advected tracer holds, the tracer front relax exponentially fast over the ridges of large Lyapunov exponents that therefore mark the front position. Another advantage of the FSLE method is that the effect of both eddies and jets are remarkably dealt with.

We have tested the barrier candidates against a synthetic tracer advected by the geostrophic field. This test sets an upper limit on the confidence on FSLE and OW assumptions, since it excludes the effect of any factor acting on the tracer except the geostrophic velocity field. At the meso- or larger scales, the OW was found to locate correctly (with errors of tens of $\mathrm{km}$ ) tracer patches when they were well confined inside eddies. Fluid parcels intruding eddies's along thin filaments are not captured however. Such filaments, as well as the submesoscale structure of tracer fronts, were found from the FSLE calculations.

In fact, the advection of a synthetic tracer can be considered itself as a direct, Lagrangian diagnostic of transport barriers. This approach however has some limitations. Lagrangian structures are sampled unevenly, since the tracer tends to spend longer times in regions with low velocities. For this reason, even strong barriers may require a carefully choice of the initial conditions and of the integration time. An example of this appears in Fig. 4, where part of the Almerian-Oran front is not entirely visible and would have required to probe the velocity field with a larger number of tracer blobs. When doing the tracer experiment of

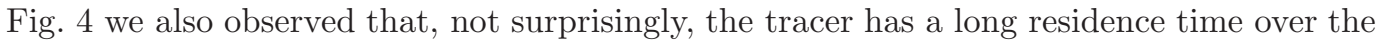
Balearic Abyssal Plain and therefore for long integration times most of the time the tracer shades the barrier of this region only, independently of its initial condition. On the other hand, a reduction of the integration time increases the gap between the tracer front and the actual barrier position, since the tracer front has a shorter time to relax to it (see the discussion of Eq. 31). In fact, an attempt to overcome these limitations requires the use of backward trajectories (for which barriers are regions of maximal separation) and of variable integration times, basically yielding a FSLE-type calculation. For this reason, the FSLE can be considered an optimised way of advecting a passive tracer for the detection of transport structures. Note also that, besides guaranteeing a uniform sampling of the velocity field and the precise localization of transport barriers, the FSLE also provides the information of the intensity of the barrier at the same cost of tracer advection.

The representation of advection in the Algerian basin however is based on altimetry and therefore is limited by the lack of representation of any non-geostrophic component of the velocity field as well as unresolved or poorly resolved structures due to space and time resolution (Pascual et al., 2006), and noise. The comparison with SST images allows to verify that such effects are not strong enough to spoil the FSLE usefulness in locating Lagrangian features. Note that the filament tangle connecting different eddies as well as the spiral structures inside eddy cores cannot appear in any way in a time-independent field (i.e., by using a snapshot of the velocity field), where one can show that orbits correspond to altimetric contours, and eddy barriers to closed contours. Nevertheless, the slow time variability of a few altimetric frames is enough to generate Lagrangian structures which correspond well to SST images. We also expected an effect of altimetric errors especially for the structures close to the coast, but with the exception of the position of the first gyre in the Alboran sea and in spite of the chaotic nature of the geostrophic advection, the localization of features from Lyapunov exponent maps appears quite reliable.

Climatologies of several Eulerian diagnostics and either barrier densities or average values 
of Lyapunov exponents also showed distinct different patterns. Given the fact that the main differences between Lyapunov and Eulerian diagnostics are related to small scale filaments and in the temporal variability of mesoscale turbulence, it is not difficult to expect different relation between Eulerian diagnostics and Lyapunov exponents computed at much larger scale and on shorter time periods. This is the case of the $0.5 \mathrm{deg}$. and 15 days calculation of in Waugh et al. (2006) (resp. 50 times larger and 3 times shorter than in our case). We illustrate this in Fig. 11, where the Lyapunov exponents have been computed with initial separations of $0.5 \mathrm{deg}$. for the same day of Fig. 3 $\mathrm{k}$. Indeed, at the resolution of the Mediterranean altimetry product (1/8 deg.) not even the patterns of two Eulerian diagnostics like the strain and the eddy kinetic energy appear to be correlated (Fig. $9 \mathrm{~b}$ and c). An interesting exercise would be the calculation of correlation between eddy kinetic energy and Lyapunov exponents computed at various scales, in order to see whether there exists a critical lengthscale or a smooth transition. In fact, the finite-size Lyapunov exponent has been introduced in turbulence theory with the aim of probing a velocity field at various scales. General arguments (Aurell et al., 1997) suggest the presence of a cross-over scale in the Lyapunov calculation separating an exponential regime from other diffusive regimes at the scale of the smallest resolved eddy. For the case of altimetry, this cross-over can be expected to appear at spatial scales of the order of $1 \mathrm{deg}$.

Note that the Lyapunov exponent is not an advanced replacement of Eulerian diagnostics, but a complementary tool incorporating tracer information. For instance, the association of lobes with eddies that in some cases we observed only holds for stationary eddies. If used systematically as a Lagrangian eddy detection, this approach would lead to detect spurious, small-scale spatial signal, that in fact comes from the temporal variability of large-scale eddies. The OW and FSLE method are also complementary from a technical viewpoint. The OW method is a differential technique. It is thus affected by small-scale noise and especially by discontinuities. These kind of features indeed can appear in the presence of eddies with a size close to the spatial resolution. On the contrary, the FSLEs are computed by integrating particle trajectories and thus are not strongly affected by local noise or discontinuities. The complementarity of the information detected by FSLE in respect to the OW parameter and other Eulerian diagnostics is also emphasised in the climatologies of Fig. 7 that showed how the differences detected in single day analyses are indeed a persistent feature.

The main drawbacks of the FSLEs are a relatively increased complexity in the calculation (with respect to the OW) and especially the need of extended velocity data sets, both in time and in space. This can be a problem if the observational region is limited (either in time or space), since some trajectories may go out from the boundaries (this is in fact at the basis of other methods used to characterize flow structures (Schneider et al., 2005)).

The OW on the contrary can be calculated from a snapshot of the velocity field. Indeed, its easiness allows to compute it even from in situ data (e.g. Stocker and Imberger, 2003; Testor and Gascard, 2005; Pallás and Víudez, 2005) and then obtain a first approach to the flow topology and its effect on the transport. Furthermore, although OW is a differential technique and therefore more sensible to velocity errors or discontinuities, it is possible to approach it using kinetic energy at least for the identification of vortex core edges (Paparella et al., 1997; Isern-Fontanet et al., 2004). If the vortex is assumed to be close to axial symmetry then the OW formula can be written as

$$
W \propto \frac{1}{r}\left(\frac{\partial E(r)}{\partial r}\right),
$$

where $r$ is the radius from the vortex center and $E(r)$ its density of kinetic energy at that distance from the center. This implies that the line of $W=0$ will correspond to a local 
maximum of energy.

The comparison with SST and synthetic tracer patterns also shows that the errors in the OW method and the FSLE technique are of very different nature. The OW fails with both the real and synthetic tracer mainly for detection of the filamentation process, due to its intrinsic lack of representation of the time variability of the velocity field. In contrast, the FSLE is limited not by its assumption of time-scale separation between barrier and tracer, but because of the limits of the altimetric data. This is clearly shown by the fact that the FSLE performs almost ideally for the synthetic case while only providing a more qualitative estimations of SST fronts. In perspective, envisaging the availability of assimilation and observational velocities field of increased quality and spatiotemporal resolution, one can expect a decrease in effectiveness of the OW technique in favour of the FSLE (or any other Lagrangian technique) at least for the detection of (sub-)mesoscale transport barriers.

\section{Conclusions}

The advantages of a Lagrangian tool when compared to Eulerian diagnostics in capturing tracer structures would be trivial for model data. What is not trivial is that altimetry data are amenable to such a comparison, given that the differences arise in presence of tracer filaments, i.e., at a scale similar or even smaller than the nominal resolution of altimetry data (and much smaller than the cutoff in the altimetry energy spectrum). At this scale ageostrophic components (unresolved by altimetry data) as well as SST active dynamics is supposed to play an important role. The fact that neverthelss altimetry-derived patterns approach SST structures means that these unresolved components of the flow are either of weak intensity or act in phase with the horizontal stirring (Lehahn et al., 2007). The recent availability of realistic, submesoscale resolving biogeochemical models (e.g. (Resplandy et al., 2008)) should give the possibility of exploring the role of unresolved ageostrophic components and of tracer activity on filament detection.

The fact that altimetry data can be pushed at subgrid scales when re-analised by Lagrangian tools, providing systematic information of filament location, opens several interesting perspectives. For the case of high-resolution biogeochemical measurements, the Lyapunov exponent provides an unpaired tool helping to interpret the measurements in terms of the precise position of mesoscale and sub-mesoscale fronts. The use of Lyapunov calculation can be even concieved in real-time, for targetting frontal structures during in-situ surveys. This possibility comes from the fact that the Lyapunov calculation only requires the past history of the velocity field, and from the availability of altimetry products with 1-2 weeks of delays (e.g. from AVISO).

A second interesting possibility is the use of the Lyapunov exponent together with highresolution tracer measurments for the validation of a velocity field. An improved altimetry product (as what is expected from the recent launch of Jason-2 and incoming altimetry satellites) should bring a velocity field at better spatial and temporal resolution. The possibility of computing with Lyapunov calculation the position of fronts and of comparing them with high-resolution SST or chlorophyll images is one of the very few possibilities for testing the ability of the new products of better representing mesoscale and sub-mesoscale processes. This validation would be especially useful for choosing the optimal combination of spatial and temporal resolution of surface current products. A similar approach can be used also for validating assimilation products and in general for tuning the spatiotemporal resolution of circulation models. 
Given the fact that transport barriers in the ocean evolves on a much slower time scale than advected tracers, one can also approximate the short-time advection of a released tracer as a relaxation dynamics towards the stronger transport barriers and in this way estimate the tracer ditribution in the near future. The most evident application of this system would be, for example, the prediction of the evolution of pollutants. Other Lagrangian techniques have already approached this problem (Lekien et al., 2005).

\section{Acknowledgments}

This work is a contribution to the FISICOS (FIS2007-60327) project of the Spanish MEC and PIF project OCEANTECH of the Spanish CSIC. FdO has been supported by MarieCurie grant 024717-DEMETRA and CNES. JIF has been supported by Marie-Curie grant 041476-OCEAN3D. The altimeter products were produced by Ssalto/Duacs and distributed by Aviso, with support from CNES (http://www.aviso.oceanobs.com). AVHRR data were obtained from the SAIDIN database at ICM (http://ers.cmima.csic.es/saidin/). We acknowledge the help received from Marie-Héléne Rio, Alejandro Morales and Isabelle TaupierLétage.

\section{References}

Abraham, E., 1998. The generation of plankton patchiness by turbulent stirring. Nature $391(5), 577-580$.

Abraham, E., Bowen, M., 2002. Chaotic stirring by a mesoscale surface-ocean flow. Chaos $12,373-381$.

Abraham, E. R., Law, C. S., Boyd, P. W., Lavender, S. J., Maldonado, M. T., Bowie, A., 2000. Importance of stirring in the development of an iron-fertilized phytoplankton bloom. Nature 407, 727-730.

Artale, V., Boffetta, G., Celani, A., Cencini, M., Vulpiani, A., 1997. Dispersion of passive tracers in closed basins: Beyond the diffusion coefficient. Phys. Fluids 9, 3162-3171.

Aurell, E., Boffetta, G., Crisanti, A., Paladin, G., Vulpiani, A., 1997. Predictability in the large: an extension of the Lyapunov exponent. J. Phys. A 30, 1-26.

Basdevant, C., Philipovitch, T., 1994. On the validity of the "Weiss criterion" in twodimensional turbulence. Physica D 113, 17-30.

Boffetta, G., Lacorata, G., Redaelli, G., Vulpiani, A., 2001. Detecting barriers to transport: a review of different techniques. Physica D 159, 58-70.

Bouzinac, C., Vázquez, J., Font, J., 1998. CEOF analysis of ERS-1 and TOPEX/POSSEIDON combined altimetric data in the region of Algerian current. J. Geophys. Res. 103, 8059-8071.

Boyd, P., Watson, A. J., Law, C. S., et al., 2000. A mesoscale phytoplankton bloom in the polar southern ocean stimulated by iron fertilization. Nature 407, 695-702.

Bracco, A., LaCasce, J., Pasquero, C., Provenzale, A., 2000. The velocity distribution of barotropic turbulence. Phys. Fluids 12 (10), 2478-2488.

Bradbury, I., Snelgrove, V. R., 2001. Contrasting larval transport in demersal fish and benthic invertebrates: The roles of behaviour and advective processes in determining spatial pattern. Can. J. Fish. Aquatic Sci. 58, 811-823.

Brasseur, P., Beckers, J. M., Brankart, J., Shoenauen, R., 1996. Seasonal temperatures and salinity fields in the Mediterranean sea: climatologycal analysis of a historical data set. Deep-Sea Res. I 43, 159-192. 
Chaigneau, A., Pizarro, O., 2005. Eddy characteristics in the eastern south pacific. J. Geophys. Res. 110, C06005.

Coulliette, C., Wiggins, S., 2001. Intergyre transport in a wind-driven, quasigeostrophic double gyre: An application of lobe dynamics. Nonlin. Proc. in Geophys. 8, 69-94.

d'Ovidio, F., Fernández, V., Hernández-García, E., López, C., 2004. Mixing structures in the mediterranean sea from finite-size lyapunov exponents. Geophys. Res. Lett. 31, L17203.

Elhmaïdi, D., Provenzale, A., Babiano, A., 1993. Elementary topology of two-dimensional turbulence from a Lagrangian viewpoint and single-particle dispersion. J. Fluid Mech. $257,533-558$.

Font, J., Isern-Fontanet, J., Salas, J., 2004. Tracking a big anticyclonic eddy in the Algerian basin (Western Mediterranean Sea). Sci. Mar. 68 (3), 331-342.

García-Olivares, A., Isern-Fontanet, J., García-Ladona, E., 2005. Dispersion of passive tracers and Finite-Scale Lyapunov Exponents in the Western Mediterranean sea. Deep-Sea Res. II 54, 253-268.

Haller, G., 2001. Distinguished material surfaces and coherent structures in threedimensional fluid flows. Physica D 149, 248-277.

Haller, G., Yuan, G., 2000. Lagrangian coherent structures and mixing in two-dimensional turbulence. Physica D 147, 352-370.

Hua, B., Klein, P., 1998. An exact criterion for the stirring properties of nearly twodimensional turbulence. Physica D 113, 98-110.

Hua, B., McWillams, J., Klein, P., 1998. Lagrangian accelerations in geostrophic turbulence. J. Fluid Mech. 366, 87-108.

Isern-Fontanet, J., Font, J., Garcia-Ladona, E., Emelianov, M., Millot, C.and TaupierLetage, I., 2004. Spatial structure of anticyclonic eddies in the Algerian basin (Mediterranean Sea) analyzed using the Okubo-Weiss parameter. Deep-Sea Res. II 51, 3009-3028.

Isern-Fontanet, J., García-Ladona, Font, J., 2003. Identification of marine eddies from altimetry. J. Atmos. Oceanic Technol. 20, 772-778.

Isern-Fontanet, J., García-Ladona, Font, J., 2006. The vortices of the Mediterranean sea: an altimetric viewpoint. J. Phys. Oceanogr. 36 (1), 87-103.

Iudicone, D., Lacorata, G., Rupolo, V., Santoleri, R., Vulpiani, A., 2002. Sensitivity of numerical tracer trajectories to uncertainties in OGCM velocity fields. Ocean Modelling 4 (3-4), 313-325.

Jeong, J., Hussain, F., 1995. On the identification of a vortex. J. Fluid Mech. 285, 69-94.

Joseph, B., Legras, B., 2002. Relation between kinematic boundaries, stirring and barriers for the Antartic Polar Vortex. J. Atmos. Sci. 59, 1198-1212.

Koh, T., Legras, B., 2002. Hyperbolic lines and the stratospheric polar vortex. Chaos 12 (2), 382-394.

LaCasce, J., Ohlmann, C., 2003. Relative dispersion at the surface of th Gulf of Mexico. J. Mar. Res. 61 (3), 285-312.

Lacorata, G., Aurell, E., Vulpiani, A., 2001. Drifter dispersion in the adriatic sea: Lagrangian data and chaotic model. Ann. Geophysicae 19, 121-129.

Lapeyre, G., Klein, P., Hua, B., 1999. Does the tracer gradient vector align with strain eigenvectors in 2d turbulence? Phys. Fluids 11 (12), 3729-3737.

Lapeyre, G., Klein, P., Hua, B. L., 2006. Oceanic restratification forced by surface frontogenesis. J. Phys. Oceanogr. In press.

Larnicol, G., Ayoub, N., Le Traon, P., 2002. Major changes in the Mediterranean sea level variability from 7 years of TOPEX/POSEIDON ERS-1/2 data. J. Mar. Syst. 33-34, 63-89.

Le Traon, P., Nadal, F., Ducet, N., 1998. An improved mapping method of multisatellite altimeter data. J. Atmos. Oceanic Technol. 15, 522-534.

Legal, C., Klein, P., Treguier, A.-M., Paillet, J., 2006. Diagnostics of the vertical motions in a mesoscale stirring region. J. Phys. Oceanogr. 37, 1413-1424.

Lehahn, Y., d'Ovidio, F., Lévy, M., Heyfetz, E., 2007. Stirring of the Northeast Atlantic spring bloom: a lagrangian analysis based on multi-satellite data. J. Geophys. Res. 112, 
C08005.

Lekien, F., Coulliette, C., Mariano, A. J., Ryan, E. H., Shay, L. K., Haller, G., Marsden, J., 2005. Pollution release tied to invariant manifolds: A case study for the coast of Florida. Physica D 210, 1-20.

Lévy, M., Klein, P., Treguier, A.-M., 2001. Impact of sub-mesoscale physics on production and subduction of phytoplankton in an oligotrophic regime. J. Mar. Res. 59, 535-565.

Lévy, M., Lehahn, Y., Andre, J.-M., Mémery, L., Loisel, H., Heifetz, E., 2005. Production regimes in the Northeast Atlantic: A study based on SeaWiFS chlorophyll and OGCM mixed layer depth. J. Geophys. Res. 110, 10.1029/2004JC002771.

López, C., Neufeld, Z., Hernández-García, E., Haynes, P., 2001. Chaotic advection of reacting substances: Plankton dynamics on a meandering jet. Phys. Chem. Earth B 26, 313-317.

Mancho, A., Small, D., Wiggins, S., 2006. A tutorial on dynamical systems concepts applied to Lagrangian transport in oceanic flows defined as finite time data sets. Phys. Rep. 437, $55-124$.

Martin, A., 2003. Phytoplankton patchiness: the role of lateral stirring and mixing. Progress in Oceanography 57, 125-174.

Martin, A., Richards, K. J., Bracco, A., Provenzale, A., 2002. Patchy productivity in the open ocean. Global. Biogeochem. Cycles 16, 10.1029/2001GB001449.

McGillicuddy, D. J., Robinson, A. R., Siegal, D. A., Jannasch, H. W., Johnson, R., Dicky, T. D., Mcneil, J., Michaels, A. F., Knap, A. H., 1998. Influence of mesoscale eddies on new production in the Sargasso Sea. Nature 394, 263-265.

Miller, P., Jones, C., Rogerson, A., Pratt, L., 1997. Quantifying transport in numerically generated velocity fields. Physica D 110, 105-122.

Millot, C., 1999. Circulation in the western Mediterranean sea. J. Mar. Syst. 20, 423-442.

Millot, C., Benzohra, M., Taupier-Letage, I., 1997. Circulation off algeria infered from the médiprod-5 current meters. Deep-Sea Res. I 44, 1467-1495.

Morrow, R., Birol, F., Griffin, D., Sudre, J., 2004. Divergent pathways of cyclonic and anti-cyclonic ocean eddies. Geophys. Res. Lett. 31, L24311.

Okubo, A., 1970. Horitzontal dispersion of floatable particles in the vicinity of velocity singularities such as convergences. Deep-Sea Res. 17, 445-454.

Ovchinikov, I., 1966. Circulation in the surface and intermediate layers of the Mediterranean. Oceanology 6, 48-59.

Paci, A., Caniaux, G., Gavart, M., Giordani, H., Lévy, M., Prieur, L., Reverdin, G., 2005. A high resolution simulation of the ocean during the POMME experiment. part 1 : simulation results and comparison with observations. J. Geophys. Res. 110, 10.1029/2004JC002712.

Pallás, E., Víudez, A., 2005. Diagnosing mesoscale vertical motion from horizontal velocity and density data. J. Phys. Oceanogr. 35, 1744-1762.

Paparella, F., Babiano, A. Basdevant, C., Provenzale, A., Tanga, P., 1997. A Lagrangian study of the Antartic polar vortex. J. Geophys. Res. 102 (D6), 6765-6773.

Pascual, A., Faugere, Y., Larnicol, G., Le Traon, P., 2006. Improved description of the ocean mesoscale variability by combining four satellite altimeters. Geophys. Res. Lett. 33, L02611, doi:10.1029/2005GL024633.

Pasquero, C., Provenzale, A., Babiano, A., 2001. Parametritzation of dispersion in twodimensional turbulence. J. Fluid Mech. 439, 279-303.

Pierrehumbert, R., 1991. Large-scale horizontal mixing in planetary atmospheres. Phys. Fluids A 3 (5), 1250-1260.

Puillat, I., Taupier-Letage, I., Millot, C., 2002. Algerian eddies lifetime can near 3 years. J. Mar. Syst. 31, 245-259.

Resplandy, L., Lévy, M., d'Ovidio, F., Marlivat, L., 2008. Evidence of submesoscale dynamics in pCO2 sequestration. Submitted to Global Biogeochem. Cycles .

Ridderinkhof, H., Zimmerman, J., 1992. Chaotic stirring in a tidal system. Science 258, $1107-1111$. 
a)

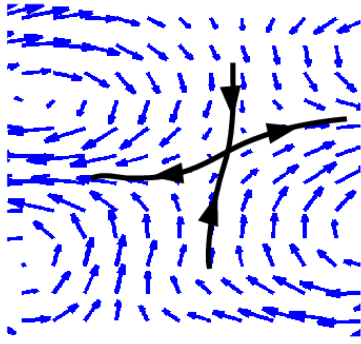

b)

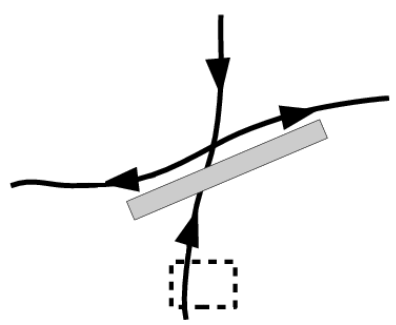

Fig. 1. A sketch of a hyperbolic structure (a) and its effect on an tracer advected by the velocity field and initialised in the dashed region (b). The unstable manifold acts as a transport barrier and generates a front, while the stable manifold cannot be directly compared with the tracer distribution. A more quantitative picture is presented in Fig. 4b.

Rio, M.-H., Poulain, P.-M., Pascual, A., Mauri, E., Larnicol, G., 2007. A mean dynamic topography of the Mediterranean sea computed from altimetric data and in-situ measurements. J. Mar. Syst. 65, 484-508.

Robinson, A. R. et al., 1993. Mesoscale and upper ocean variabilities during the 1989 JGOFS bloom study. Deep-Sea Res. II 40, 9-35.

Rossi, V., López, C., Sudre, J., E., H.-G., Garçon, V., 2008. Comparative study of mixing and biological activity of the Benguela and Canary upwelling systems. Geophys. Res. Lett. 35, L11602, doi:10.1029/2008GL033610.

Sallee, J. B., Speer, K., Morrow, R. M., Lumpkin, R., 2007. An estimate of Lagrangian eddy statistics and diffusion in the mixed layer of the southern ocean. Submitted to Journal of Climate Submitted.

Sallee, J. B., Wienders, K., Speer, K., Morrow, R. M., 2006. Formation of subantarctic mode water in the southeastern Indian Ocean. Ocean Dynamics 56, 525-542.

Schneider, J., Fernández, V., Hernández-García, E., 2005. Leaking method approach to surface transport in the Mediterranean Sea from a numerical ocean model. J. Mar. Syst. $57,111-126$.

Shadden, S. C., Lekien, F., Marsden, J. E., 2005. Definition and properties of Lagrangian structures from finite-time Lyapunov exponents in two-dimensional aperiodic flows. Physica D 212, 271-304.

SSALTO/DUACS User Handbook, January 2006. (M)SLA and (M)ADT Near-Real Time and Delayed Time Products. CLS-DOS-NT-06.034.

Stocker, R., Imberger, J., 2003. Horizontal transport and dispersion in the surface layer of a medium-sized lake. Limnol. Oceanogr. 48, 971-982.

Testor, P., Gascard, J.-C., 2005. Large-scale flow separation and mesoscale eddy formation in Algerian basin. Progress in Oceanography 66, 211-230.

Toner, M., Kirwan Jr, A., Poje, A., Kantha, L., Muller-Karger, F., Jones, C., 2003. Chlorophyll dispersal by eddy-eddy interactions in the gulf of mexico. J. Geophys. Res. 108, 10.1029/2002JC001499.

Vignudelli, S., 1997. Potential use of ERS-1 an Topex/Posseidon altimeters for resolving oceanographic patterns in the Algerian Basin. Geophys. Res. Lett. 24 (14), 1787-1790.

Waugh, D., Abraham, E., Bowen, M., 2006. Spatial variations of stirring in the surface ocean: A case study of the Tasman sea. J. Phys. Oceanogr. 36 (3), 526-542.

Weiss, J., 1991. The dynamics of enstrophy transfer in two-dimensinal hydrodynamics. Physica D 48, 273-294.

Young, W. R., Rhines, P. B., Garrett, C. J. R., 1982. Shear-flow dispersion, internal waves and horizontal mixing in the ocean. J. Phys. Oceanogr. 12, 515-527. 

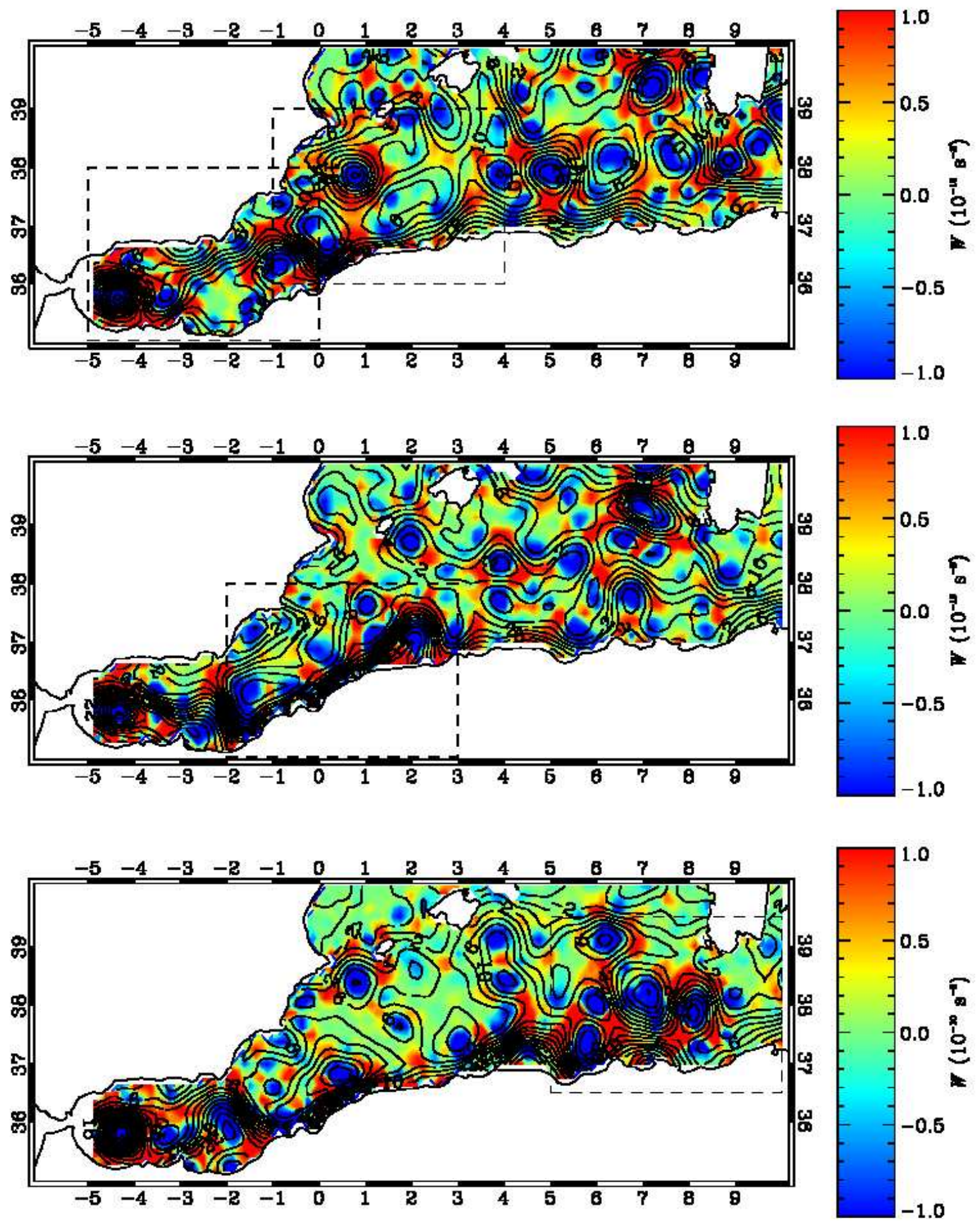

Fig. 2. Okubo-Weiss parameter ( $W$, coded in colors) computed from sea-surface height (black line) fields corresponding (from top to bottom) to July 9, 2003; April 7, 2004; June 30, 2004. The dashed boxes represent the regions over which we perform a comparison with SST (see Figs. 5 and [6). 

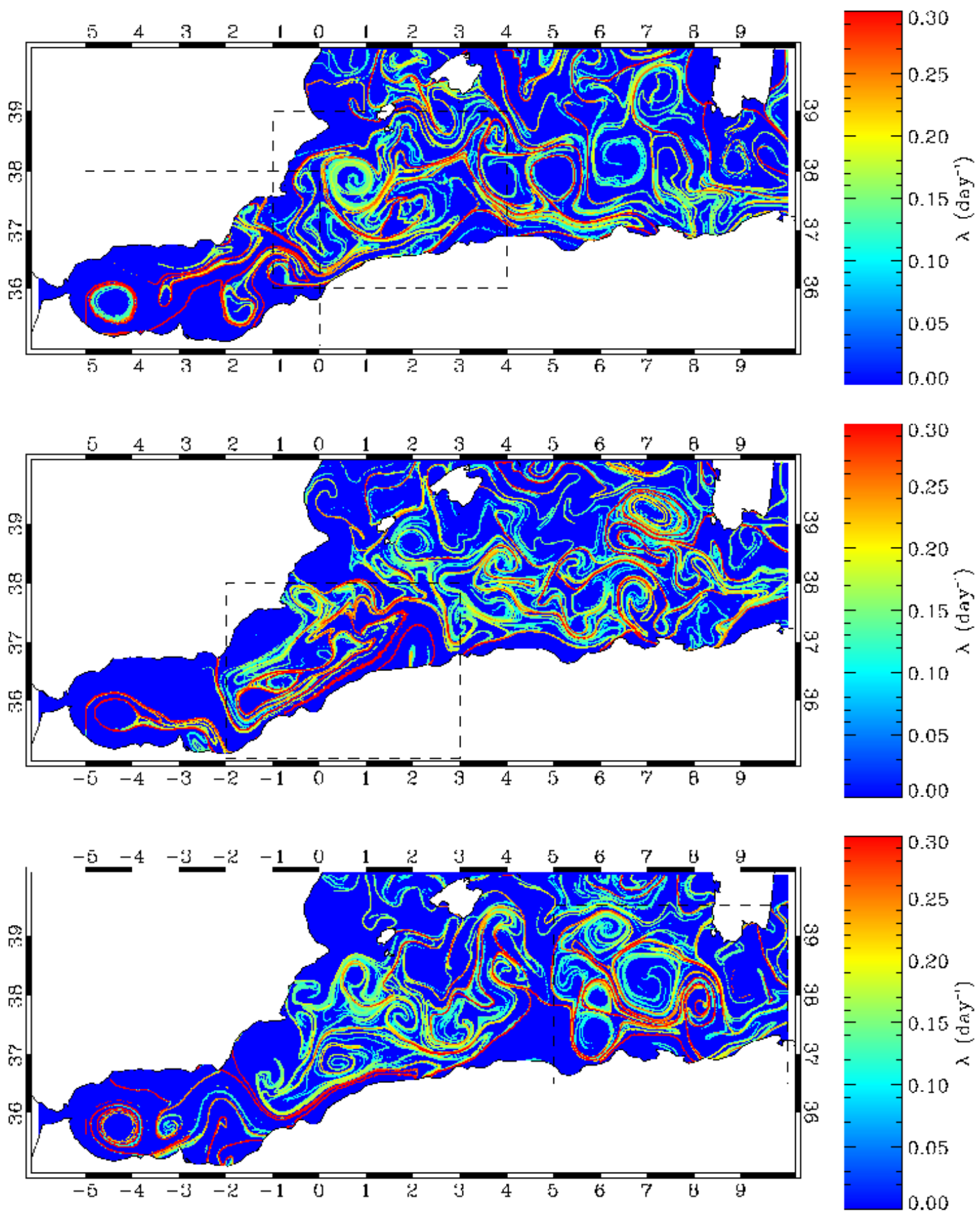

Fig. 3. FSLE $(\lambda)$ computed from sea-surface height fields corresponding (from top to bottom) to July 9, 2003; April 7, 2004; June 30, 2004. The dashed boxes represent the regions over which we perform a comparison with SST (see Fig. [5). 

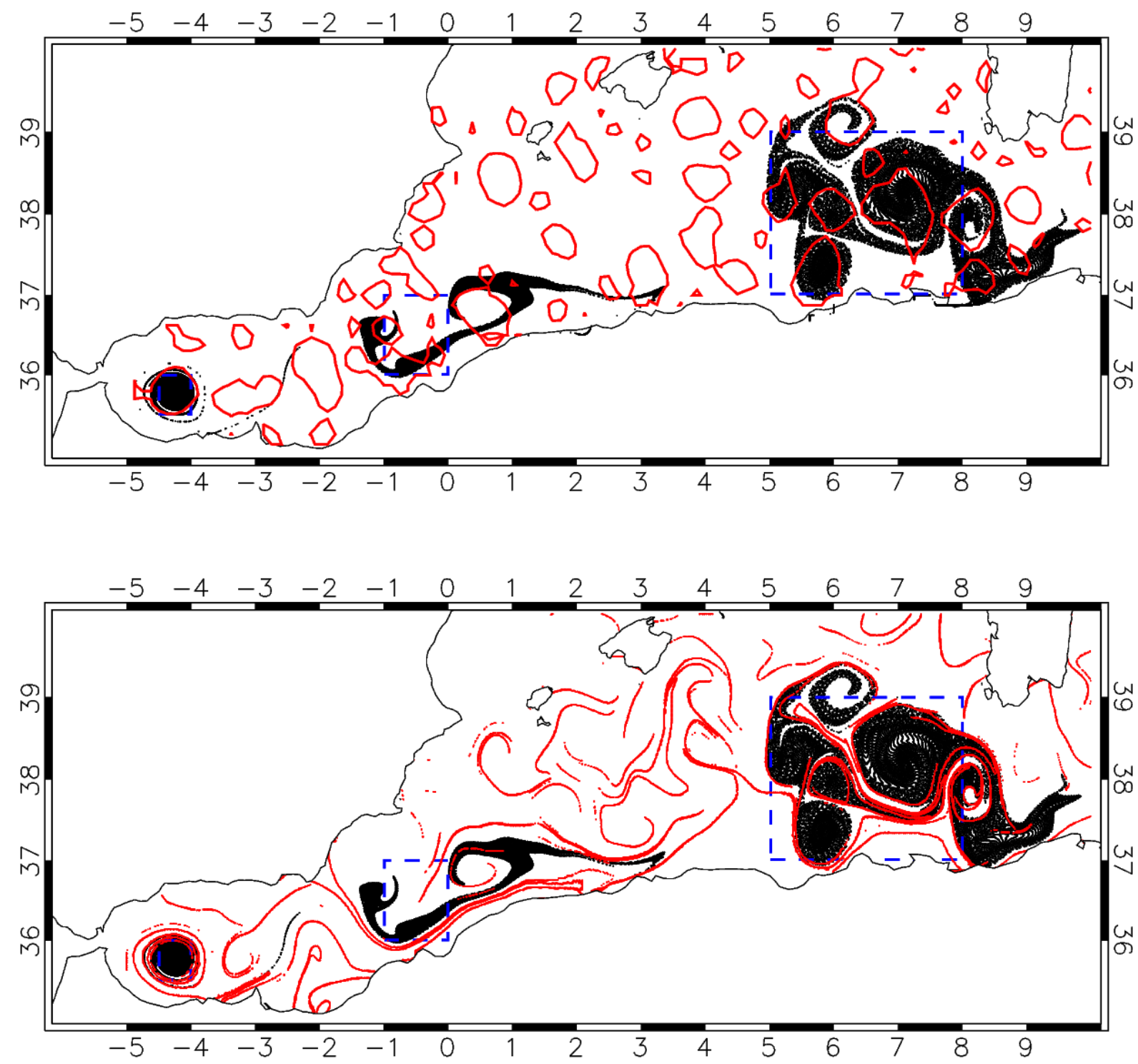

Fig. 4. Distribution of tracer particles released over the Algerian current, the western Alboran eddy, and the easternmost part of the Algerian basin (dotted boxes). Tracers in the Algerian current are advected for one week, and the other two sets are advected for two weeks. The release dates are chosen to have for the three cases the coinciding final time (June 30, 2004) which is shown in the figure. Zero-lines of OW corresponding to the final day are overimposed (top) as well as the line-shaped regions where the backwards FSLE values are larger than 0.2 days $^{-1}$ (bottom), approximating unstable manifolds of the chaotic flow. 

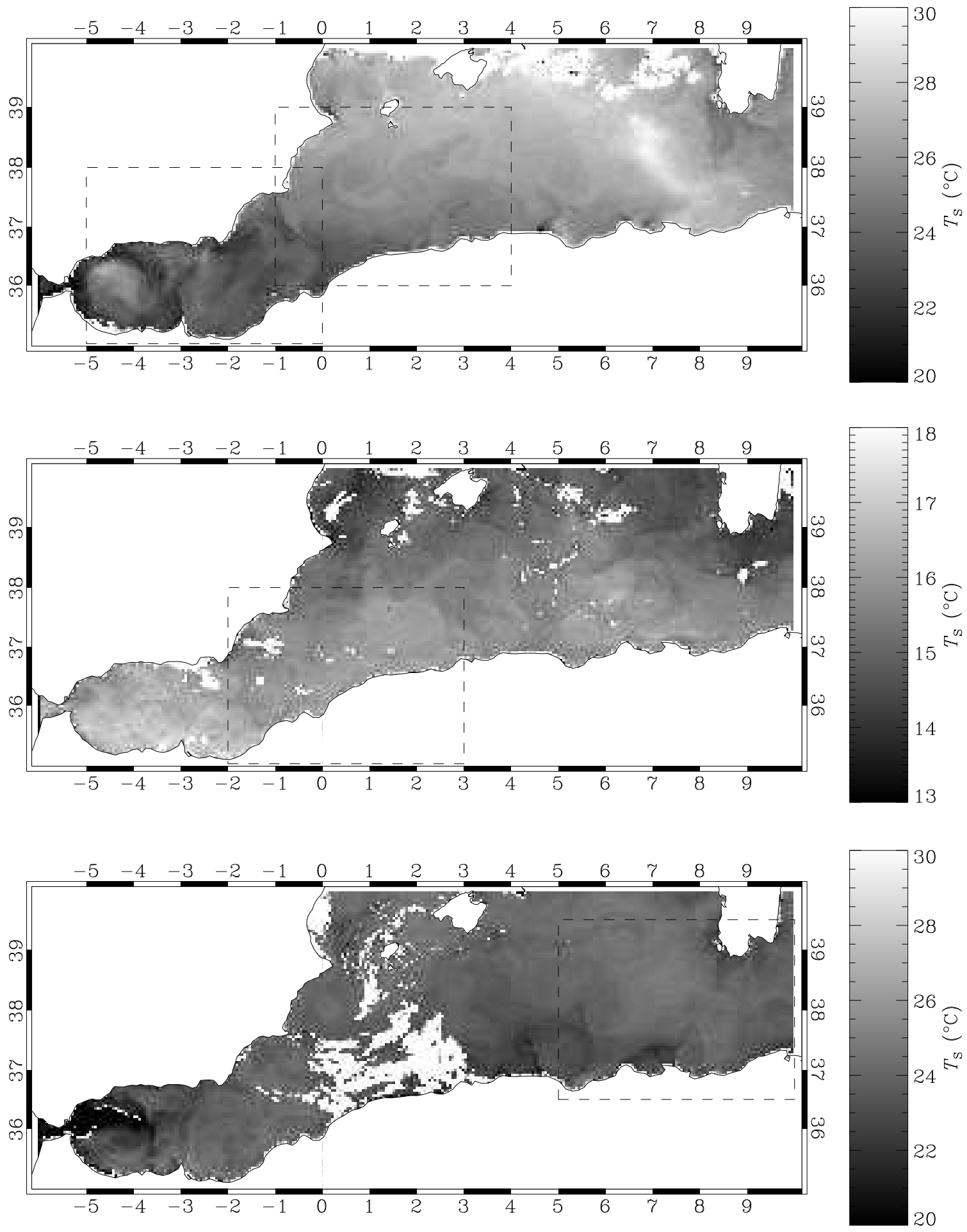

Fig. 5. General views of the sea-surface temperature fields corresponding (from top to bottom) to July 9, 2003; April 7, 2004; June 30, 2004. 

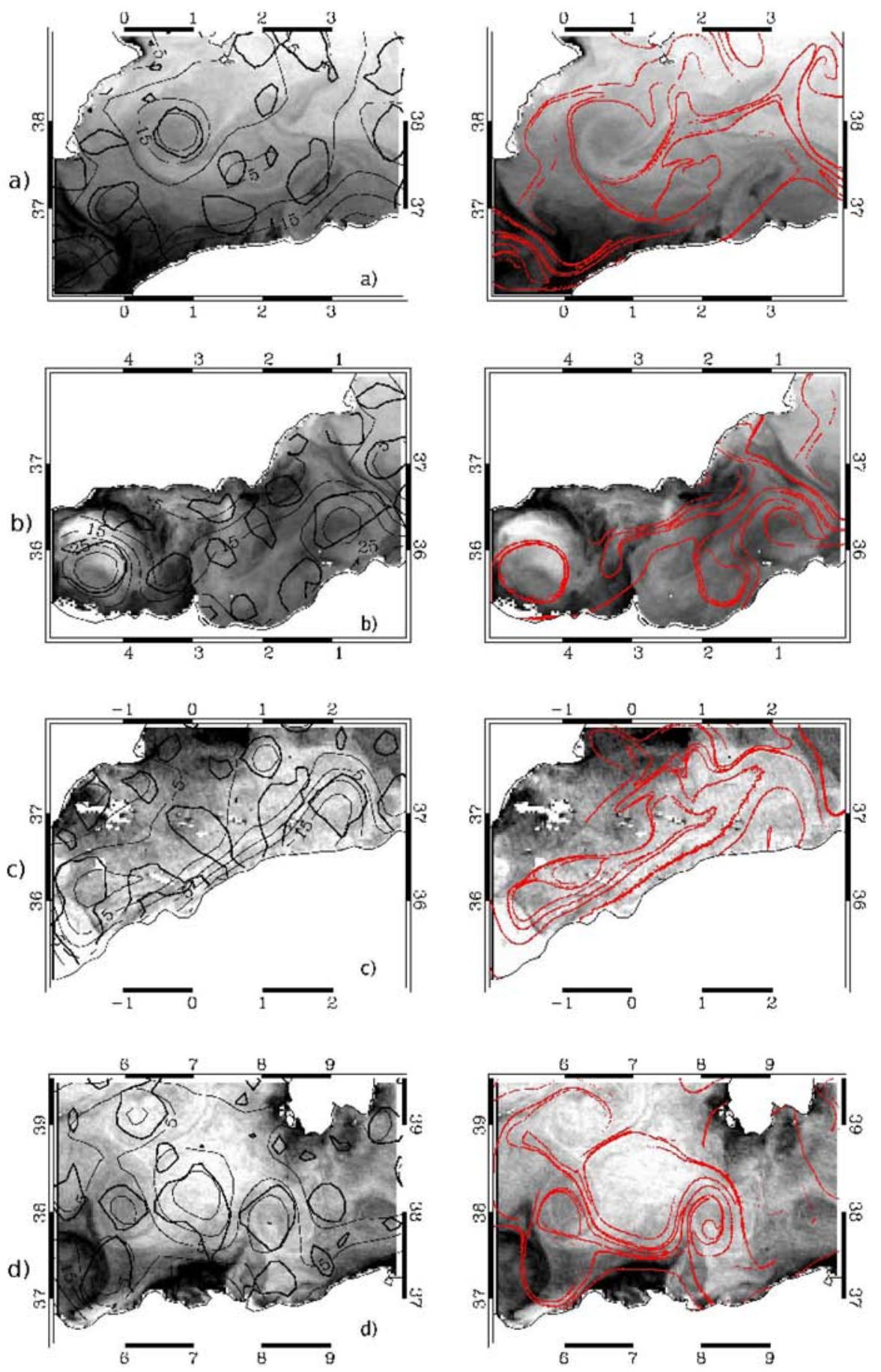

Fig. 6. Comparison of OW, FSLE and temperature distribution corresponding to the regions shown in figures 2 25. Left column: SST (coded in grey levels) with SSH (thin black line) and lines of zero OW (thick black line). Right column: SST (in grey levels) with regions where FSLE is greater than 0.1 day $^{-1}$ (red line-shaped regions). From top to bottom the dates are: July 9, 2003 (a and b); April 7, 2004 (c) and July 2, 2004 (d). 

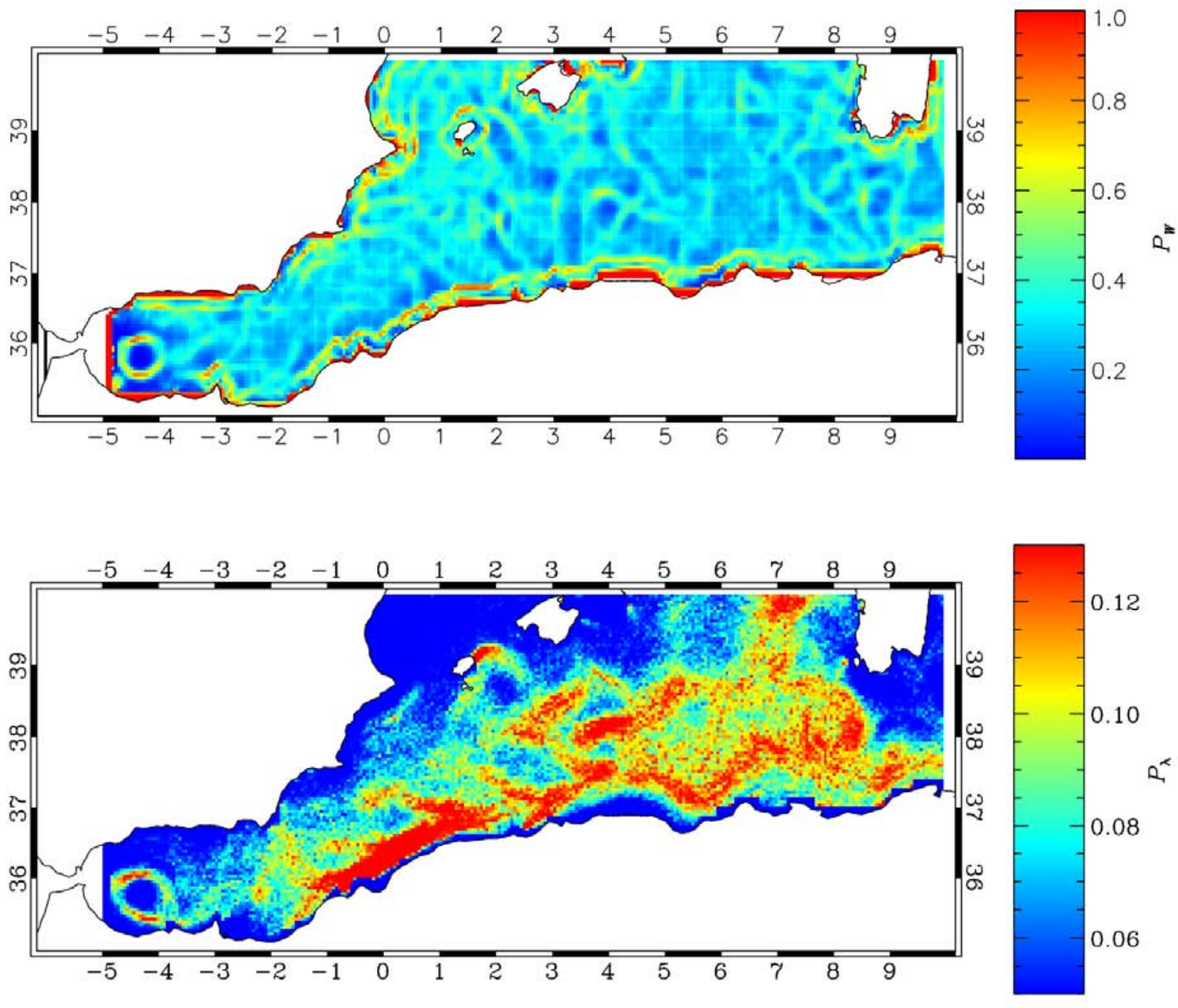

Fig. 7. Fraction of time during which each spatial point is visited by the lines $W=0$ $\left(P_{W}\right.$, upper panel) and by the ridges (local maxima along some direction) of FSLE $\left(P_{\lambda}\right.$, lower panel) for the period 1994-2004. 


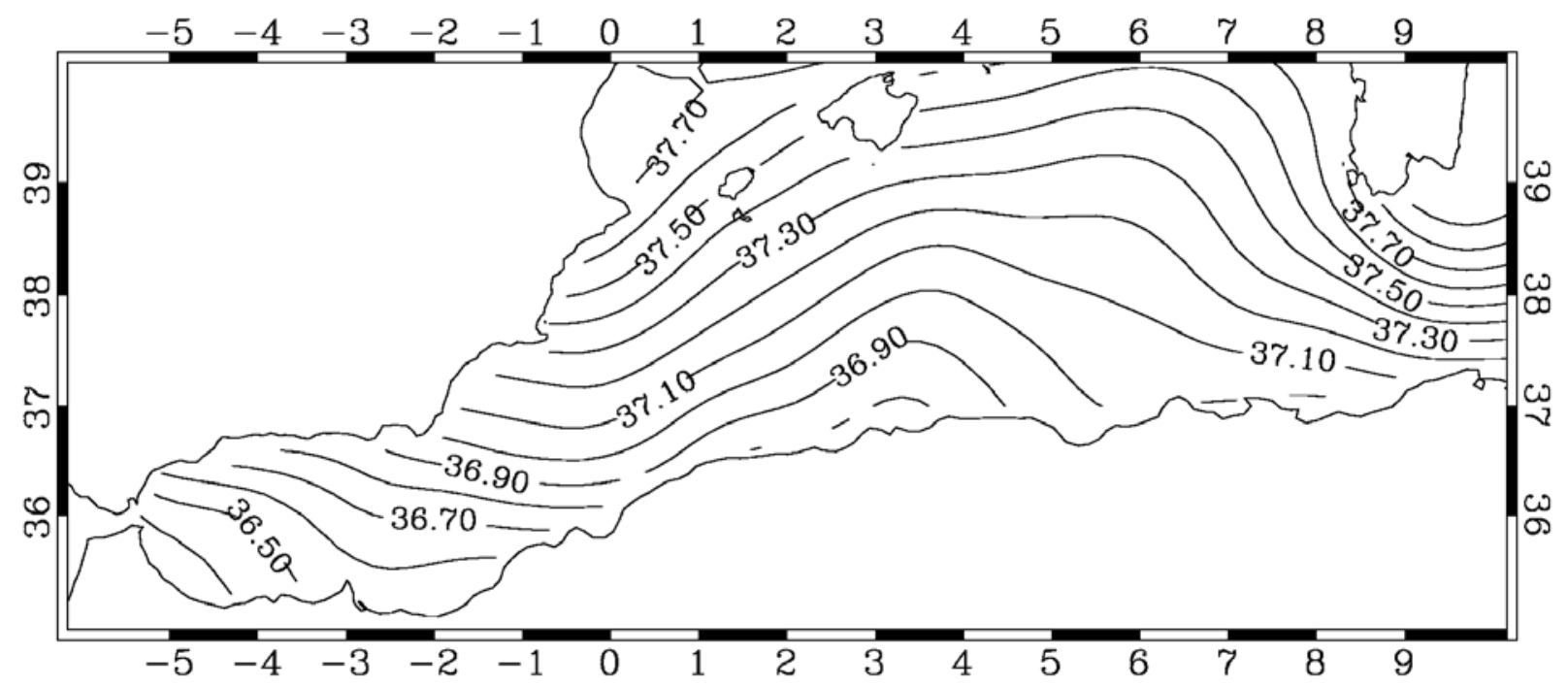

Fig. 8. Climatological distribution of salinity in the area of study at $5 \mathrm{~m}$ depth from the MEDATLAS-II data set. 

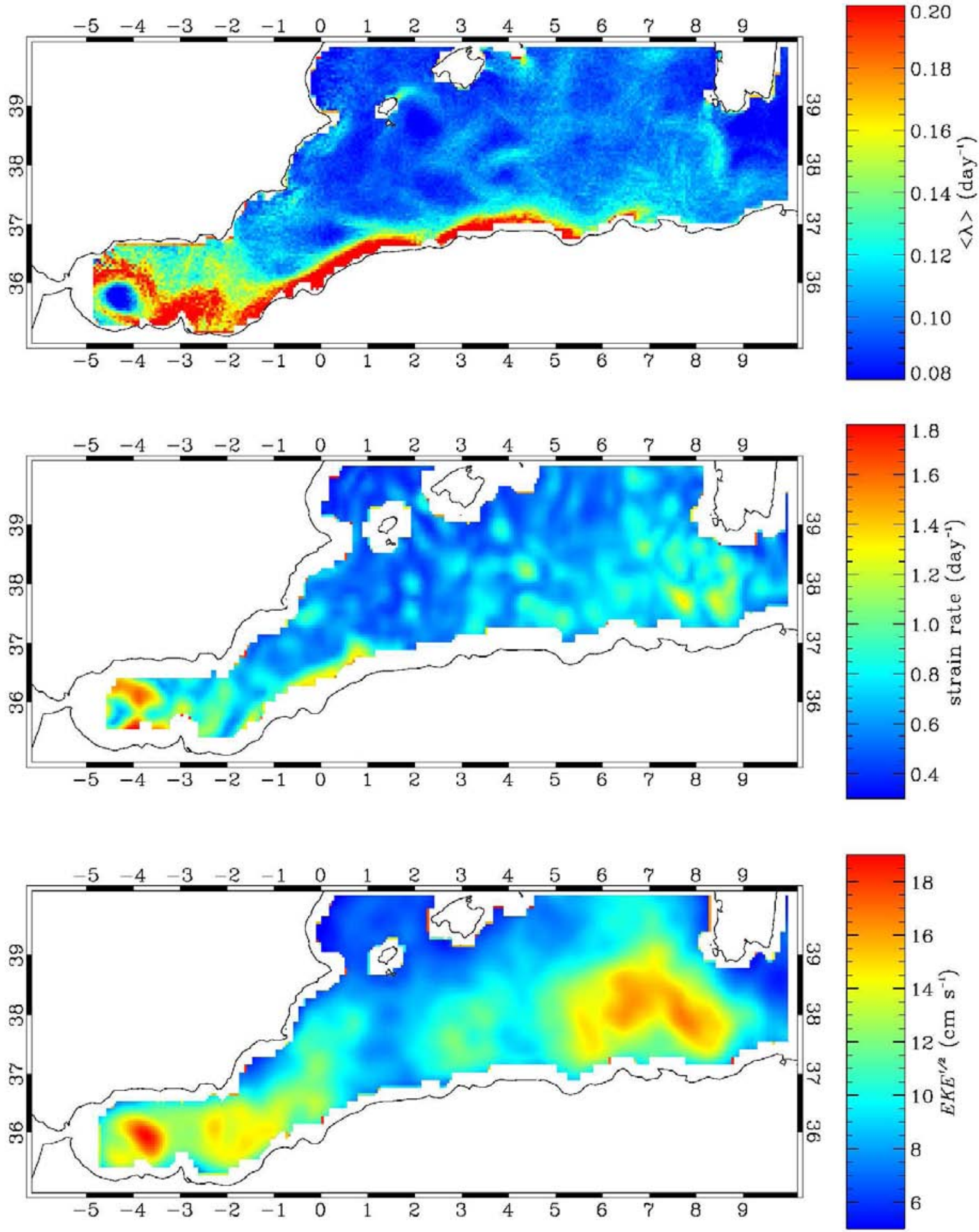

Fig. 9. Time-averaged FSLE, strain rate and eddy kinetic energy (1994-2004). 

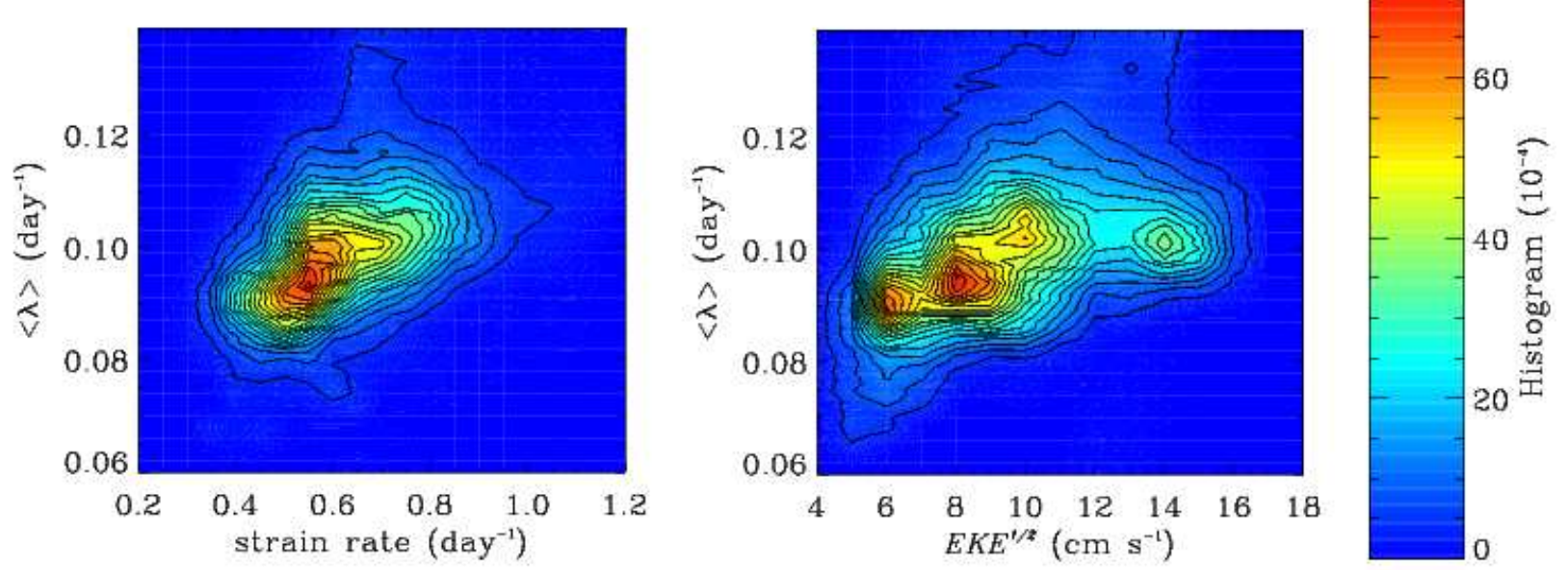

Fig. 10. Joint distributions of Finite-size Lyapunov exponents vs. strain rate values (left), and Finite-size Lyapunov exponents vs. eddy kinetic (right) from the spatial distributions time-averaged over the period 1994-2004.

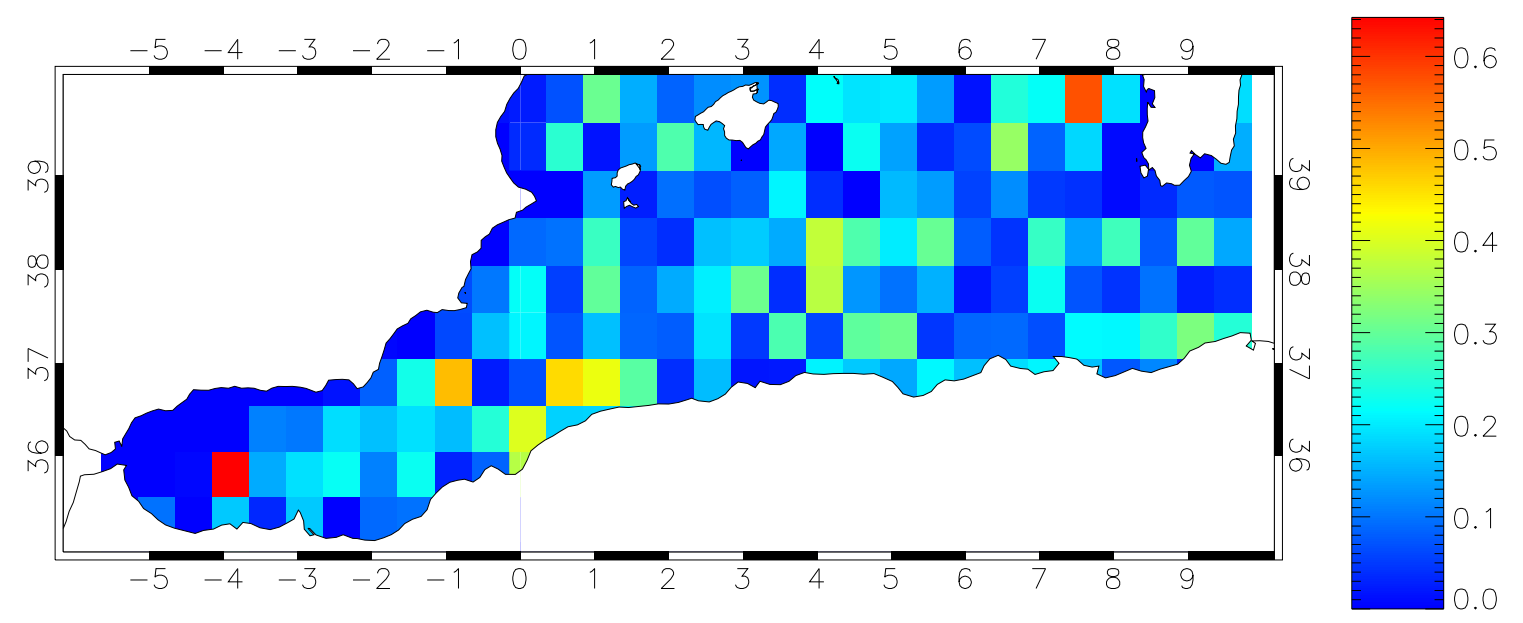

Fig. 11. Calculation of the FSLE for July 9, 2003 with initial separation $\delta_{0}=0.5^{\circ}$. Compare with Fig. 3 a. At this resolution, Lyapunov exponents cannot be linked to tracer filaments. 